This item was submitted to Loughborough's Research Repository by the author.

Items in Figshare are protected by copyright, with all rights reserved, unless otherwise indicated.

\title{
Spreading of trisiloxanes over thin aqueous layers
}

PLEASE CITE THE PUBLISHED VERSION

https://doi.org/10.1134/S1061933X09030119

PUBLISHER

(c) Pleiades Publishing (distributed by Springer Science+Business Media LLC.)

VERSION

AM (Accepted Manuscript)

LICENCE

CC BY-NC-ND 4.0

REPOSITORY RECORD

Lee, Khai S., Victor Starov, T.J.P. Muchatuta, and S.I.R. Srikantha. 2009. "Spreading of Trisiloxanes over Thin Aqueous Layers". figshare. https://hdl.handle.net/2134/4911. 
This item was submitted to Loughborough's Institutional Repository (https://dspace.lboro.ac.uk/) by the author and is made available under the following Creative Commons Licence conditions.

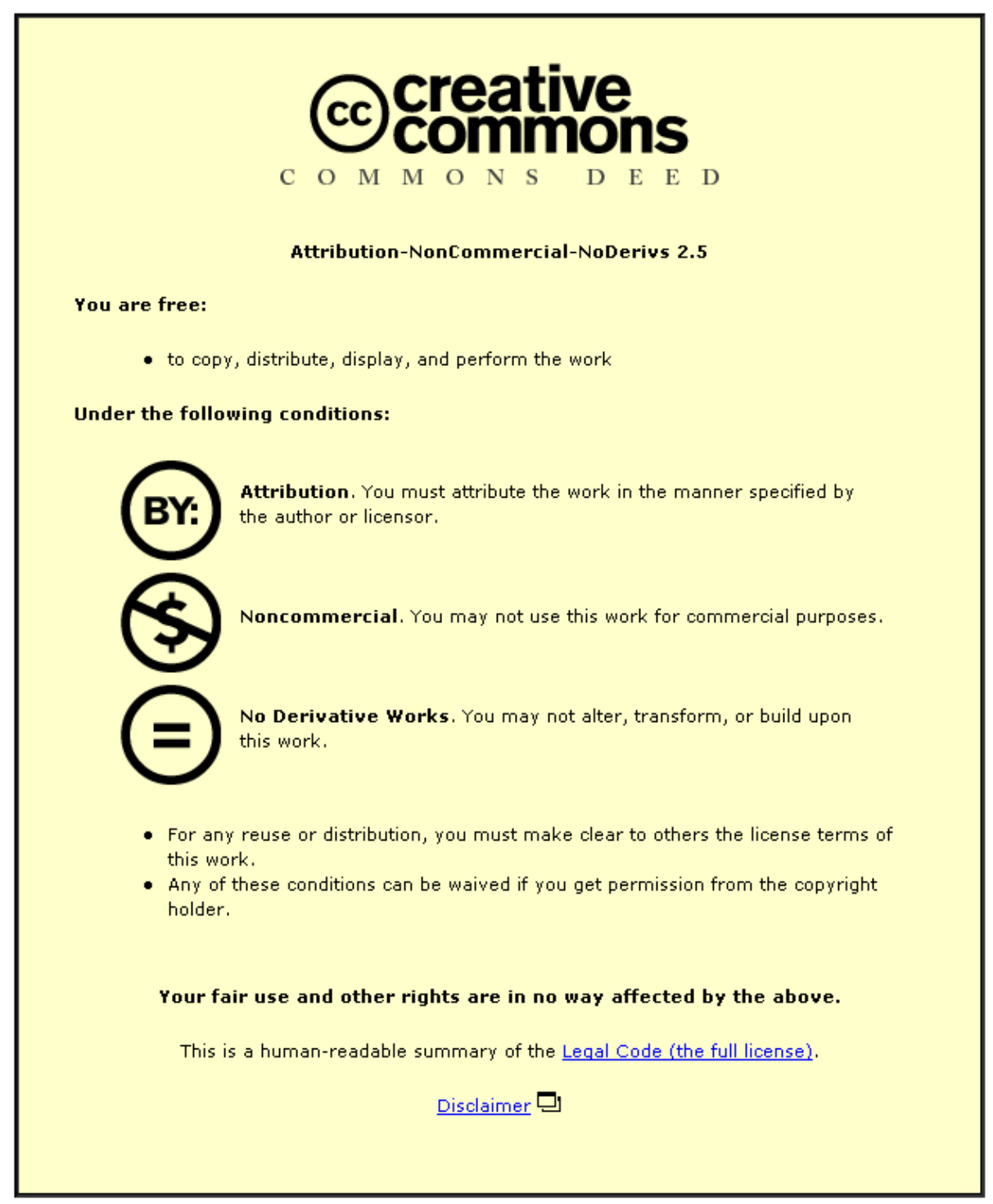

For the full text of this licence, please go to: http://creativecommons.org/licenses/by-nc-nd/2.5/ 


\title{
Spreading of trisiloxanes over thin aqueous layers
}

\author{
K. S. Lee, V. M. Starov, T. J. P. Muchatuta, S. I. R. Srikantha \\ Department of Chemical Engineering, Loughborough University, \\ Loughborough, LE11 3TU, UK
}

\begin{abstract}
Surfactants are widely spread in nature and are increasingly used in industry as wetting, cleaning and disinfecting agents. Recently, there are newly discovered trisiloxane and other silicone based surfactants which show very unusual spreading behaviour. Although a number of experimental and theoretical investigations have been carried out, the underlying spreading mechanism remains unclear. Experiments using trisiloxanes and comparison with 3 different ethylene glycol monododecyl ethers (C12E4, C12E5, and C12E6) surfactants were performed to understand the influence of Marangoni force as the driving force for the spreading. We then compared our experimental results to available theoretical prediction in the literature. The obtained experimental data showed the opposite trend as compared with the theoretical predictions developed for a regular surfactants. The latter is assumed to be a special feature of "suprspreaders".
\end{abstract}


Keywords: trisiloxane, surfactant solutions, Marangoni effect.

\section{Introduction}

Thin liquid films can be found in many engineering, geology, and biophysics environment. Their applications are significant in many coating processes [1,2,3] and physiological applications [4]. Presence of nonuniform temperature or surface active compounds across thin liquid films will lead to formation of shear stresses, also known as Marangoni gradients at the air-liquid interface. These gradients cause mass transfer on, or in a liquid layer due to surface tension non-uniformity. Marangoni stresses distribute the liquid from areas of low surface tension to areas of high surface tension (flow generation) and in doing so also deform the interface resulting in height variations (deformation and instability of liquid films). In this article, we shall restrict our discussions to surfactant induced Marangoni flows only.

The understanding of Marangoni induced flows is important as it can be beneficial and detrimental to many applications. Surfactants which are normally present in a healthy mammalian lung to reduce surface tension forces, which keeps the lungs compliant and prevents collapse of the small airways during exhalation. However, most prematurely born babies does 
not produce adequate amount of these surfactants which leads to respiratory distress syndrome (RDS). This condition is treated by surfactant replacement therapy (SRT) where surfactants are introduced into the lungs. These surfactants spread by gravitational forces in the large to medium pulmonary airways. In small airways where gravity is negligible, surface tension gradients dominates and Marangoni flow distributes the surfactant to the distal regions of the lung [4]. A common problem in coating processes where paint films are dried by solvent evaporation is that the non-uniformity of the evaporation leads to Marangoni stresses which cause deformation in the film and hence, permanent defects on the paint surface. Another common application of the Marangoni effect is the use for drying silicon wafers after a wet processing step during the manufacturing of integrated circuits.

A type of silicone surfactant, trisiloxanes, was found to possess an unusual ability to induce wetting on hydrophobic surfaces. This phenomenon was termed as superspreading [8-9]. When added to water, trisiloxane surfactants are capable of lowering the surface tension from 70 to about 20 dyn $\mathrm{cm}^{-1}$.

Fig. 1 depicts the general molecular structure of a trisiloxane. Commonly, a shorthand notation is used for the trisiloxane surfactants, in which these surfactants are denoted $M\left(D^{\prime} E n\right) M$ where $M$ stands for the trimethylsiloxy 


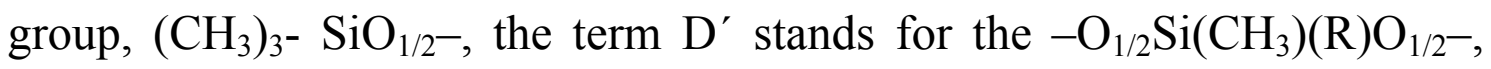
where $\mathrm{R}$ is a polyoxyethylene group attached to the silicon by way of a propyl spacer, and En stands for polyoxyethylene,- $\left(\mathrm{CH}_{2}-\mathrm{CH}_{2} \mathrm{O}\right) n \mathrm{R}-$. However, for simplicity, for example, a trisiloxane with 8 polyoxyethylene group will be termed T8.

Recent developments in the superspreading behaviour have been summarised in [5]. In spite of a number of experimental investigations the underlying mechanism of superspreading is still not revealed and at present there is no understanding of the mechanism of superspreading. It is a real challenge to understand this mechanism, which will open new perspectives for both chemical engineering and colloid science.

In superspreading experiments several forces are involved, which tend to spread drops of superspreading surfactant solution over hydrophobic substrates. These forces are: gravity force, which becomes dominant for "big" drops; capillary force, which is dominant for "small" drops; Marangoni force, caused by surface tension gradient on the drop surface; surface forces, which arose in thin layers close to the three phase contact line. A number of experimental observations on the spreading of aqueous trisiloxane drops over flat hydrophobic surfaces were performed for the investigation of the kinetics of spreading $[6,7,8,9]$. However, in all these investigations sufficiently big drops were used which mean that gravity, 
capillary, Marangoni and surface forces acted simultaneously and it was impossible to separate them from each other in such experiments. The latter observation means that it is difficult to use the previous experimental data for revealing the mechanism of superspreading. Therefore it is necessary to find a way to separate the influence of these acting forces. Below we use a method, which allows investigating the Marangony forces separately.

Spreading of aqueous trisiloxane surfactant solution under sole Marangoni influence was conducted using trisiloxanes varying from $3-9$ polyoxyethylene groups and 3 different ethylene glycol monododecyl ethers (C12E4, C12E5, and C12E6). An attempt was made to justify the influence of Marangoni as a driving force in spontaneous spreading with reference to the currently available theory.

\section{Theory}

It has been shown earlier [10] that investigation of a motion of a front after deposition of a small droplet of aqueous surfactant solution on a thin aqueous layer allows investigating separately the influence of Marangoni phenomenon on the hydrodynamic flow because the capillary and gravitational forces can be neglected (see Fig. 2). The suggested method results in a stable motion of the wetting front. The latter allows extracting properties of surfactants on a liquid-air interface. 
Starov et. al. [10] reported theoretical and experimental work for surfactants spreading over a thin liquid layer. The authors [10] proposed a theoretical model where the spreading was subdivided in two stages; a rapid first stage and a slower second stage.

For the first stage the time dependency of radius is $R(t) \sim t^{0.5}$ and the second stage $R(t) \sim t^{0.25}$. Experimental results of aqueous sodium dodecyl sulphate (SDS) at concentration 5 times the critical micelle concentration (CMC) shows the first stage to be $0.60 \pm 0.15$ and the second stage to be $0.17 \pm 0.02$. Obtained experimental results compared relatively well with the theoretical prediction.

Lee et. al. [11] made significant changes to the previous theoretical models by taking the influence of solubility into account as this was assumed to be negligible in [10]. Surfactant droplets with concentration above CMC were deposited on thin aqueous layers in [11]. Further theoretical development revealed that surfactants' solubility significantly influences the spreading exponents (Fig. 3).

For an insoluble surfactant (a), the first stage would proceed with $R(t) \sim t^{0.75}$ and the second stage to be $R(t) \sim t^{0.25}$. For highly soluble surfactant, (c), the first stage gives $R(t) \sim t^{0.5}$ and the second stage would reach a limiting position of $R_{\infty}$. 
Lee et. al. [11] further strengthened their proposed theoretical model by investigating surfactants Tergitol NP10 (highly insoluble), SDS (moderately soluble) and DTAB (highly soluble). Shown in Fig. 4, the highly insoluble surfactant has the highest spreading exponents close to the theoretically predicted 0.75 and the highly soluble surfactant spreads during the first stage and reaches a limiting position in the second stage.

\section{Materials}

Surfactants used in the experiments were 3 different ethylene glycol monododecyl ethers (C12E4, C12E5, and C12E6) were purchased from Sigma-Aldrich UK, and 7 types of trisiloxanes (T3 - T9) were kindly supplied by Dr. Randal Hill, DowCorning US. All surfactants were used as received without further purification.

The solid substrate used in the spreading experiments is a $20 \mathrm{~cm}$ diameter borosilicate Petri dish. Aqueous solutions of these surfactants were prepared with deionised water.

\section{Methodology}

10 or $50 \mathrm{ml}$ of distilled water were used to create a uniform thin aqueous layer, which covers the bottom of the Petri dish. De-wetting of the aqueous layer does not occur because the Petri dish was washed before each 
experiment according to the following protocol. The Petri dish was cleaned by (i) soaking it in chromic acid for $50 \mathrm{~min}$, (ii) extensively rinsing with distilled and deionised water after that, and (iii) dried in an oven. The cleaning procedure was repeated after each experiment. By spreading of 10 $\mathrm{ml}$ of distilled water, a thin water layer with thickness $0.32 \mathrm{~mm}$ was deposited evenly across the surface of the Petri dish. A small amount of talc powder was homogeneously smeared over the surface of the aqueous layer to trace the motion of the liquid front under the action of surfactants. High precision $5 \mu$ Hamilton syringe (Hamilton GB Ltd., UK) was used to inject 1.5 or $3 \mu 1$ droplets of aqueous surfactant solutions on the top of the aqueous layer. A mechanical manipulator was structured to enable placement of the surfactant droplet on the thin aqueous layer whilst minimising the kinetic impact of the surfactant droplet and capillary waves on the thin aqueous film.

The entire spreading process was captured using a high speed video camera (Olympus i-Speed) at a rate of 500 frames per second. The recorded video was then analysed using Olympus i-Speed software, tracking the position of the moving front/dry spot for the spreading process. Three tracking points of the spreading front radius were measured to obtain an average. The pixel/length calibration was done using a known length. For each sample, the experiment was repeated to produce at least 5 sets of data and 
each experimental point below is averaged over 5 experimental points. No instabilities of the moving front were detected in any experimental run.

\section{Results \& Discussions}

In all experiments, we observed a circular spreading front moving from the centre to the periphery of the Petri dish. The time evolution of the radius of this spreading front, $R(t)$, was monitored, which was then plotted on a loglog coordinate system. In all cases considered, we found that the spreading process can be separated into two stages; a first fast stage and a second slower stage. The power law exponents were obtained by fitting trendlines over the experimental data with a minimal fit of $95 \%$. We assumed that the higher the CMCs of the surfactants, the more soluble the surfactants would be.

According to the theoretical prediction (Eqs (1) - (4)), the lower the solubility of the surfactant, the higher the power law exponent will be. This trend was experimentally confirmed earlier [10-11] when we used regular surfactants. However, we observe this trend to be completely opposite when compared to our experimental results on spreading of superspreaders. We deposit $1.5 \mu l$ of aqueous trisiloxanes solutions at concentration $20 \mathrm{~g} / \mathrm{l}$, which is substantially higher than Critical Wetting Concentration (CWC) 
for all trisiloxanes investigated ( see [12] and references in). The time dependency of the radius is shows in Fig. 5 and the corresponding spreading exponents tabled in Table 1.

From Fig. 5 and Table 1, we clearly see that increasing the solubility of trisiloxanes (by increasing the ethoxylate groups), the higher the spreading exponent for the first stage of spreading. Highly soluble trisiloxane T9 shows the highest spreading exponent with the rate of $0.80 \pm 0.01$ whilst the least soluble trisiloxane $\mathrm{T} 3$ gives only $0.44 \pm 0.03$. The second stage proceeds with a more or less constant rate approximately $0.11 \pm 0.02$ with the exception of $\mathrm{T} 7$ where the second stage is $0.20 \pm 0.01$.

In the next set of experiments, we investigated the deposition of $3 \mu l$ drop of ethylene glycol monododecyl ether surfactants with 4, 5 and 6 ethoxylate groups at concentrations 20 times the $\mathrm{CMC}$. The results for $\mathrm{C} 12 \mathrm{E} 4, \mathrm{C} 12 \mathrm{E} 5$, and C12E6 are plotted in Fig. 6 and the spreading rates are tables in Table 2.

We observed that C12E6 which is the most soluble surfactant shows the highest spreading rates $(0.72 \pm 0.02)$, while less soluble C12E4 shows slower spreading rates $(0.51 \pm 0.01)$ for the first stage of spreading. For the second stage of spreading, C12E4 has a spreading exponent of $0.21 \pm 0.01$ while $\mathrm{C} 12 \mathrm{E} 5$ and $\mathrm{C} 12 \mathrm{E} 6$ show spreading exponents $0.15 \pm 0.01$ and $0.16 \pm$ 0.02 respectively. 
Experiments with both trisiloxane and ethylene glycol monododecyl ether surfactants exhibit a similar trend whereby the spreading rates during the first stage increases as the solubility increases. However, the solubility does not seem to influence the spreading rates during the second stage, with the exception of both T7 and C12E4. According to theoretical predictions [11], when solubility increases, spreading rates for both the first and second stage would decrease as well. Our experimental findings revealed the possibility of another different transfer mechanism of the surfactants. We assume that bulk properties of surfactants itself influences the magnitude of the surface tension gradient driving force. Trisiloxanes were found to undergo self-assembly process at specific temperatures and concentrations where superspreading was observed. The theoretical models were developed on the basis that micelles disintegrate to provide a continuous supply of surfactants to the front edge to sustain spontaneous spreading. Therefore, existence of different types of aggregates might be significant in influencing the surfactants' transfer to the edge.

\section{Conclusions}

A moving circular spreading front was observed after a small droplet of aqueous surfactant solution was deposited onto a thin aqueous layer. The time evolution of the radius of the moving front was monitored. It is shown 
that the time evolution of the moving front proceeds in two stages: a rapid first stage and slower second stage. During the first stage, we observed as the surfactant's solubility increases, so does the spreading exponents. For the second stage, the spreading exponents does not appeared to be influenced by surfactant's solubility. These observations were in complete opposite to the behaviour of regular surfactants and the theoretical prediction where the lower the solubility, the higher the spreading rates for both the first and the second stage.

\section{Acknowledgement}

This research was supported by Engineering and Physical Sciences research Council, UK (Grant D077869/1) 


\section{References}

1. L.W. Schwartz, D.E. Weidner, R.R. Eley, Proceedings of the ACS Division of Polymeric Materials Science and Engineering 73 (1995) 490.

2. J. Patzer, J. Fuchs, E.P. Hoffer, Proc. SPIE-Int. Soc. Opt. Eng. 167 (1995) 2413.

3. H.P. Le, J. Imaging Sci. Tech. 42 (1998) 49.

4. D.L. Shapiro, In Surfactant Replacement Therapy, AR Liss, New York, 1989.

5. R.M. Hill, Superspreading. Curr. Opin. Coll. \& Int. Sc. Vol. 3 (1998) 247-254.

6. S. Zhu, W.G. Miller, L.E. Scriven, H.T. Davis, Coll. \& Surf. A: Physicochem. Eng. Aspects. Vol.90 (1994) 63-78.

7. A.D. Nikolov, D.T. Wasan, A. Chengara, K. Koczo, G.A. Policello, I. Kolossvary, Adv. Coll. Int. Sci. Vol. 98 (2002) 325-338.

8. T. Stoebe, Z. Lin, R.M. Hill, M.D. Ward, H.T. Davis, Langmuir 12 (1996) 337-344.

9. T. Stoebe, R.M. Hill, M.D. Ward, H.T. Davis, Langmuir 13 (1997) $7270-7278$. 
10.Starov, V.M., de Ryck, A., and Velarde, M.G. J. Colloid Interface Sci. 190, (1997) 104.

11.Lee, K.S., Starov, V.M., J. Coll. Int. Sci. 314 (2007) 631 - 642.

12.Natalia Ivanova, Victor Starov, Daniel Johnson, Nidal Hilal, Ramon Rubio, Randal Hill. Langmuir, submitted (2008). 


\section{Figure captions}

Fig. 1

General molecular structure of trisiloxane

Fig. 2

Spreading of surfactant solutions over a thin liquid layer

Fig. 3

Spreading behaviour prediction of 3 surfactants with varying solubility (Lee et. al. [11])

Fig. 4

Log-log plot of spreading front against time for 3 different surfactants with different solubilities (Lee et. al. [11])

Fig. 5

Log-log plot of spreading front against time for 7 different types of trisiloxanes

Fig. 6

Log-log plot of spreading front against time for ethylene glycol monododecyl ether surfactants 
Table 1.

First and second stage spreading exponents for trisiloxanes

Table 2.

First and second stage spreading exponents, surfactants' properties for ethylene glycol monododecyl ether surfactants 
Figures

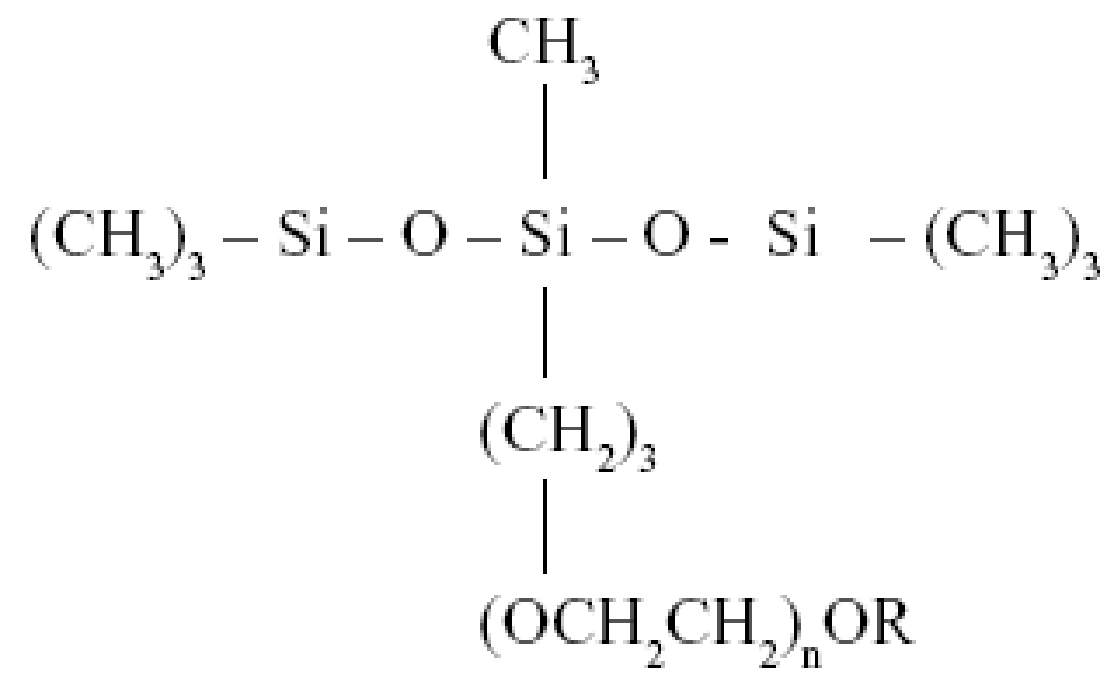

Fig. 1 
water layer

surfactant droplet

moving font

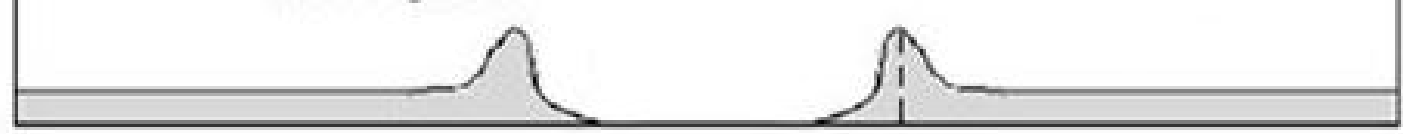

$R(t)$

Fig. 2. 


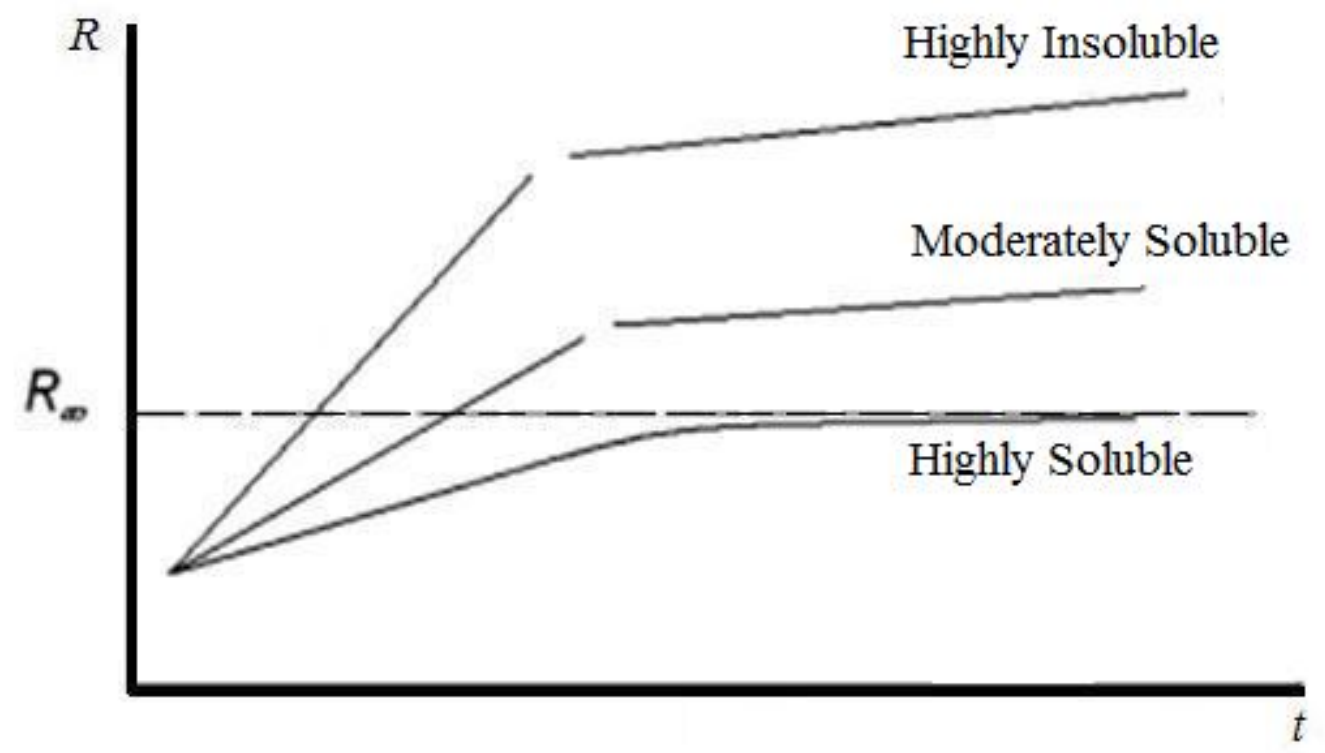

Fig. 3. 


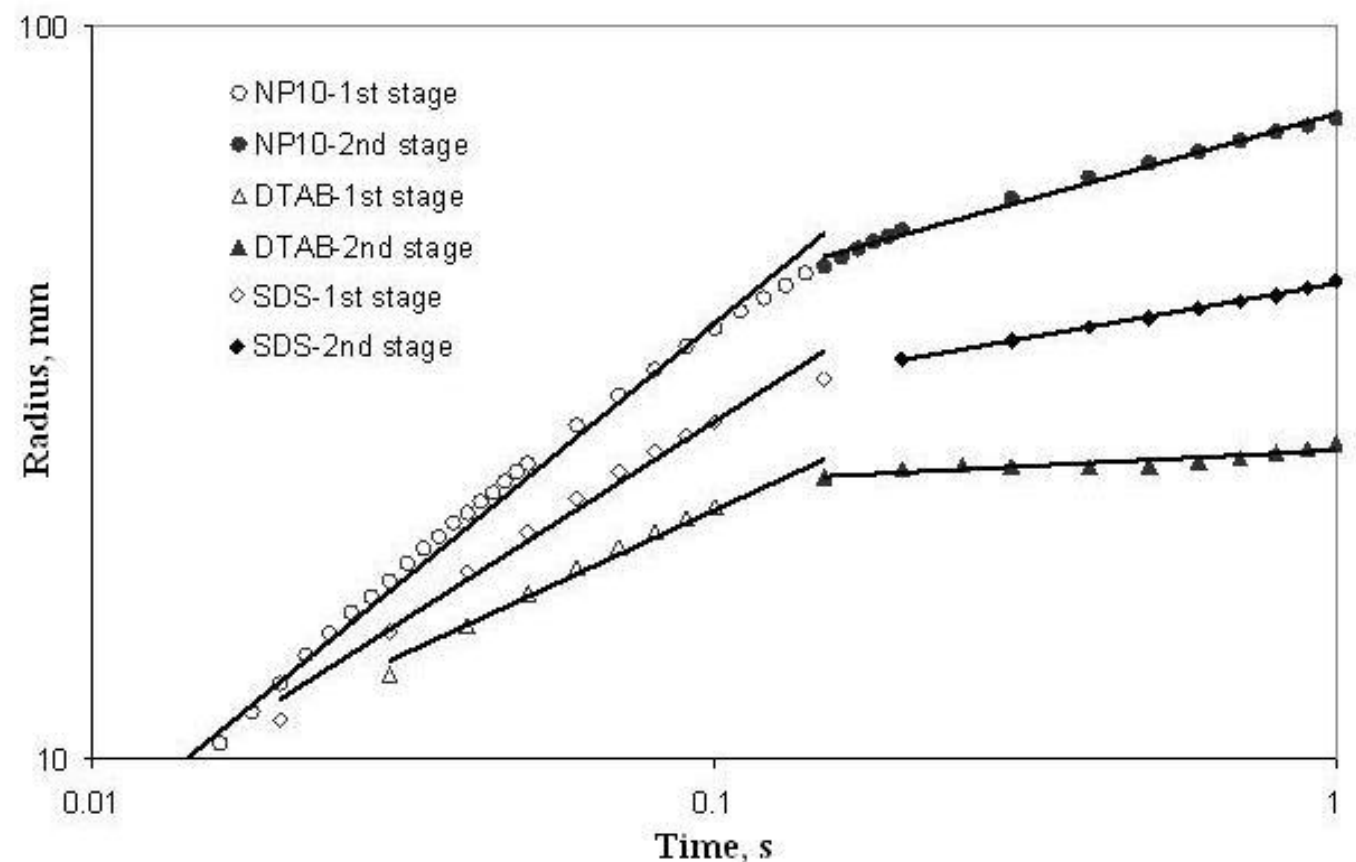

Fig. 4. 


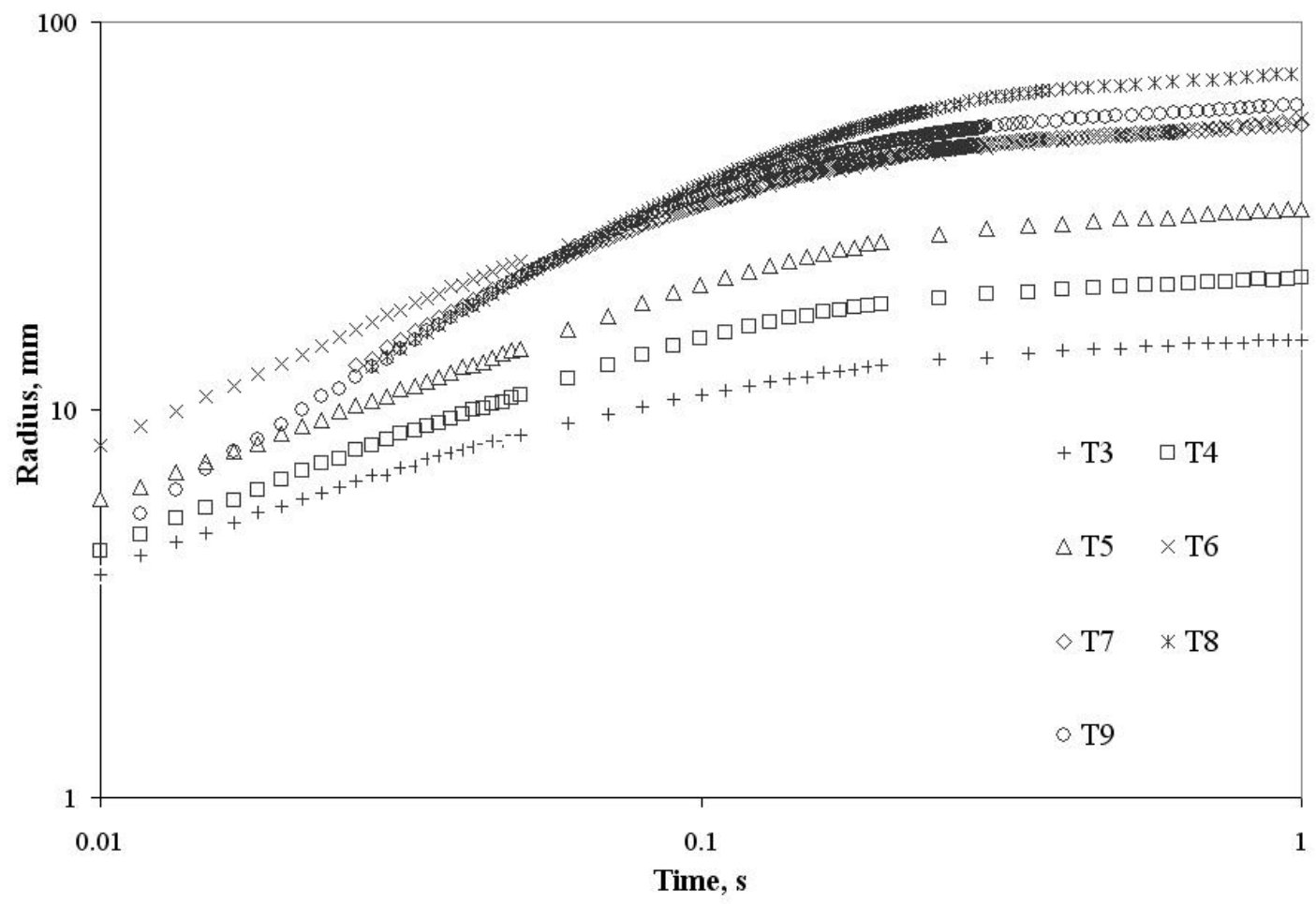

Fig. 5. 


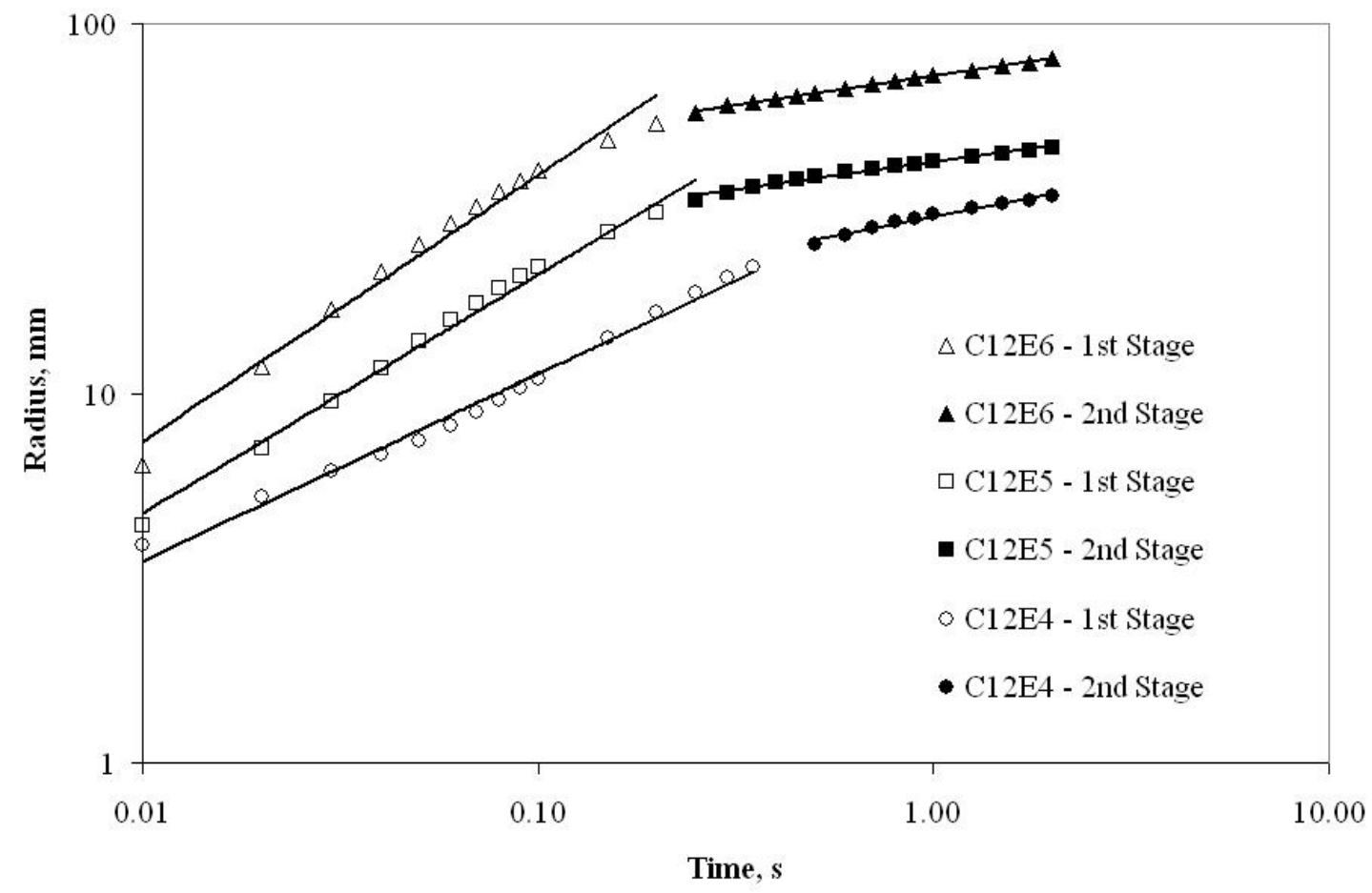

Fig. 6. 


\begin{tabular}{lccc}
\hline \multirow{2}{*}{ Materials } & Molecular weight, & \multicolumn{2}{c}{ Spreading exponent } \\
\cline { 3 - 4 } & g/mol & $1^{\text {st }}$ Stage & $2^{\text {nd }}$ Stage \\
\hline T3 & 426.86 & $0.44 \pm 0.03$ & $0.11 \pm 0.01$ \\
T4 & 470.92 & $0.52 \pm 0.01$ & $0.12 \pm 0.02$ \\
T5 & 514.98 & $0.53 \pm 0.01$ & $0.10 \pm 0.01$ \\
T6 & 559.04 & $0.63 \pm 0.02$ & $0.12 \pm 0.01$ \\
T7 & 603.10 & $0.73 \pm 0.05$ & $0.20 \pm 0.01$ \\
T8 & 647.16 & $0.76 \pm 0.02$ & $0.12 \pm 0.01$ \\
T9 & 691.22 & $0.80 \pm 0.01$ & $0.13 \pm 0.01$ \\
\hline
\end{tabular}

Table 1. 


\begin{tabular}{lcccc}
\hline \multirow{2}{*}{ Materials } & Molecular & & \multicolumn{2}{c}{ Spreading Exponent } \\
& weight, $g / m o l$ & CMC, $m M$ & & \\
\cline { 4 - 5 } & & & $1^{\text {st }}$ Stage & $2^{\text {nd }}$ Stage \\
\hline C12E4 & 362.55 & 0.050 & $0.51 \pm 0.01$ & $0.21 \pm 0.01$ \\
C12E5 & 406.61 & 0.085 & $0.65 \pm 0.01$ & $0.15 \pm 0.01$ \\
C12E6 & 450.66 & 0.087 & $0.72 \pm 0.02$ & $0.16 \pm 0.02$ \\
\hline
\end{tabular}

Table 2. 